\title{
La vie des entreprises : évaluation économique des dommages indirects aux activités
}

\author{
Companies lifes : economic evaluation of indirect damages to activities
}

par P. Desgranges

Territoires Conseil Associés

Indirect damages to activities are at first lost production; it is a major part of global damages, so it has to be precisely assessed. It must be made out a typology fonction of the flood vulnerability. For the great plants, an exhaustive inquiry is necessary to quantify the risks. The action during the crisis is important for the determination of damages amount. Risk evaluation goes through the making out of a damages curve which shows threshold effects. But not all the consequences of a great flood can be developed.

\section{I $\square$ CONCEPTS ET PROBLÉMATIQUE DE L'ÉVALUATION}

\subsection{Nature des dommages indirects}

Les dommages indirects aux activités économiques sont essentiellement constitués par la perte de valeur ajoutée durant le temps de l'interruption de la production en prenant en compte la part éventuellement transférée ou récupérée ultérieurement. Ils ont également une traduction sociale à travers le chômage technique qui peut en découler.

D'autres éléments liés à l'atteinte physique de la crue peuvent être regroupés au titre des dommages indirects : les coûts de nettoyage, des interventions d'urgence, de séchage des locaux et des équipements.

Les études françaises traitant des dommages liés aux risques d'inondation sont généralement assez pauvres quant aux analyses relatives aux dommages indirects dans le domaine des activités :

- soit les dommages indirects ne sont pas quantifiés ou non reliés aux paramètres hydrauliques,

- soit les dommages indirects sont quantifiés et reliés aux paramètres hydrauliques, mais sur la base d'une typologie d'activités très agrégée et non discriminante en termes d'endommagement.

Or les pertes d'exploitation sont d'une importance considérable dans le total des dommages indirects dans le cas des grandes crues historiques (plus de $50 \%$ du coût total pour une crue de type 1910). La démarche d'évaluation doit donc permettre une valorisation précise des dommages potentiels.

\subsection{Les démarches envisageables pour analyser le risque}

L'échelle géographique de l'étude et la précision recherchée sont les deux facteurs déterminants dans le choix de la démarche. On distinguera en schématisant deux chemins d'analyse contrastés :

a) dans une approche sommaire, les coûts indirects aux activités seront exprimés comme un pourcentage des dommages directs, variable selon la hauteur et la durée de l'inondation. Le paramétrage de ces pourcentages peut s'effectuer par analyse statistique d'autres études (données du Flood Hazard Research Centre en Grande Bretagne, par exemple).

Les imprécisions liées à cette approche sont très importantes : 1 - on "récupère" les imprécisions liées à l'estimation des dommages directs ;

2 - les différences de dommage unitaire en fonction des types d'activité ne sont pas appréhendées ; en particulier on ne différencie pas des délais de remise en route, ni des parts de production récupérable.

b) dans une approche détaillée, il sera indispensable d'enquêter auprès des entreprises situées dans la zone inondable. Le questionnaire sera établi de manière à fournir l'ensemble des éléments nécessaires à l'établissement de courbes d'endommagement et au calcul des dommages monétaires.

Dans le cas d'une zone inondable large, une enquête exhaustive peut s'avérer extrêmement lourde et coûteuse, voire impossible à réaliser matériellement. Il est alors nécessaire de hiérarchiser l'analyse en fonction des ordres de grandeur des dommages potentiels, et de concentrer les enquêtes sur les entreprises à enjeux élevés. 


\section{- 1.3 L'impossible typologie des activités}

En termes de dommages indirects, il importe de discriminer les activités par rapport aux éléments fondamentaux que sont la vulnérabilité à une hauteur et une durée de crue données. Toutefois des critères supplémentaires interviennent : ce sont la part de production récupérable et le délai de retour à la production normale, critères aggravants ou modérateurs des coûts pour une hauteur et une durée de submersion données (activité de production ou de stockage, etc.).

L'analyse de vulnérabilité s'appuie sur une description de l'impact du dommage selon trois critères :

- la sensibilité au dommage qui mesure la probabilité qu'une activité aura d'être affectée par celui-ci,

- la dépendance, qui mesure l'intensité de la perturbation induite par un dommage donné,

- la transférabilité, qui mesure la capacité d'une activité à s'adapter au dommage en transférant sa production dans l'espace ou en fournissant des solutions techniques de remplacement.

En France, les classifications INSEE permettent de partitionner les entreprises selon des nomenclatures précises : nomenclature des activités professionnelles (NAP), nomenclature des activités françaises (NAF). Le problème fondamental et récurrent est qu'il n'existe aucune homogénéité, au sein d'une catégorie donnée, en terme de caractérisation de l'endommagement. Statistiquement, on peut mettre en évidence, à partir des enquêtes réalisées, un écart-type plus fort à l'intérieur d'une catégorie qu'entre deux catégories distinctes. La désagrégation des activités en rubriques de plus en plus fines ne fait qu'accentuer ce phénomène.

Un partitionnement valide ne peut alors être envisagé qu'entre grands types d'activités tels que les activités de production, le stockage, le négoce. Les investigations auprès des experts assurantiels sont un moyen de valider la pertinence d'un calcul d'endommagement spécifique à ces types d'activités.

\section{II $\square$ LE COMPORTEMENT FACE A LA CRUE}

\subsection{L'importance du critère de comportement}

Le comportement face à la crue est une variable décisive de l'évaluation des dommages ; en effet la possibilité de préserver ou non l'appareil de production, les infrastructures et superstructures, d'organiser le redéploiement éventuel de la production, d'optimiser la gestion des ressources humaines, sont autant d'éléments considérables de variation du coût des dommages.

Dans ce schéma, l'enquête auprès des entreprises doit avoir pour vocation d'identifier le comportement face à l'inondation à partir d'hypothèses clairement formulées sur les délais d'annonces de crue, les mesures publiques envisagées, les hauteurs et débits prévisibles pour une échéance donnée. Le résultat doit être une évaluation quantifiée de la vulnérabilité de l'entreprise considérée.

\section{- 2.2 Les enquêtes sur les activités exposées}

S'agissant de la région Ile de France, la base considérable d'activités susceptibles d'être touchées par l'inondation conduit à adopter la démarche d'investigation détaillée décrite au point 1.2.

Premièrement, on identifie et on positionne les entreprises présentant un montant considérable d'enjeux exposés et pour lesquelles les crues auraient un impact économique important. On leur affectera un endommagement spécifique. Ces entreprises considérées comme stratégiques sont sélectionnées selon les critères suivants : chiffre d'affaires, nombre d'employés, en tenant compte de la vulnérabilité à l'inondation (une entreprise très importante en termes de $\mathrm{CA}$ ou d'emplois ne rentrera pas dans la base si elle n'est pas vulnérable aux crues simulées). Chaque enquête individuelle fournit une courbe d'endommagement spécifique et non destinée à être transposée.

Pour les autres activités, des courbes d'endommagement par grand type d'activités sont établies. Elles sont appliquées uniformément à chaque entreprise de la catégorie considérée de manière à pouvoir réaffecter les dommages sur une base géographique.

\section{- 2.3 Quelques résultats et conclusions provisoires}

Il ressort des enquêtes effectuées dans le cadre de l'étude d'une crue centennale type 1910 à Paris et en région Ile de France un certain nombre de constatations et de réflexions instructifs dans le cadre de l'analyse :

\section{a) les facteurs de vulnérabilité à l'inondation}

Parmi les facteurs cités par les entreprises, on retrouve le plus souvent :

- la nature du bien produit,

- la proximité de la nappe phréatique, susceptible d'induire une inondation préalablement aux débordements de surface,

- l'utilisation de l'énergie sur les sites de production : certains équipements ne peuvent être arrêtés de manière soudaine sans refroidissement préalable (activités lourdes de métaux, industrie automobile,

- l'emplacement et la hauteur des sources d'énergie et des principales machines,

- la sensibilité des produits ou du matériel à l'eau ; on retrouve ici les phénomènes de capillarité (stock de papiers pour les imprimeries), l'oxydation, les atteintes aux équipements électriques et électroniques, aux cablages,

- la dépendance au salissement et aux dégradations induites par la présence de l'eau : problème d'hygiène pour les produits pharmaceutiques par exemple,

- l'influence du système de production à flux tendus sur la vulnérabilité, en liaison notamment avec les problèmes d'accès aux sites de production dans le cas de rotations rapides des livraisons et de l'approvisionnement,

- la capacité ou non de reprise rapide de l'activité, de report ou d'étalement de la production.

\section{b) Le comportement face à la crue}

Un premier point concerne la décision de poursuite de l'activité. Cette décision est elle-même facteur de la souplesse des normes de sécurité acceptées par la direction, sur proposition de l'ingénieur sécurité-environnement.

C'est pourquoi l'arrêt de l'activité peut être décidé précocement par rapport à la montée des eaux : sera ainsi rapidement interdit l'accès aux tours des sièges sociaux, les centres de grande distribution (Hypermarchés) seront fermés dès que les normes de sécurité incendie ne sont plus respectées... d'autres agents économiques sont plus enclins à continuer 
coûte que coûte : entreprises de travaux publics habituées aux conditions dures sur les chantiers, PME...

La prévention des dommages est une autre composante du comportement face à la crue. Une différence essentielle apparaît selon qu'il est possible ou non de déplacer le matériel sensible. Dans de nombreux cas, le déplacement ou la surélévation ne sont pas envisageables (chaînes robotisées, équipements mécaniques lourds). Quand on peut envisager un déplacement ou une surélévation, un arbitrage se fera en termes de coûts respectifs des différentes solutions, incluant le "laissez-faire".

Les entrepreneurs insistent dans ce cas sur la fiabilité de l'information liée à la crue et qui leur sera transmise : importance de l'inondation (débit, hauteurs prévisibles), délais d'anticipation, probabilités d'atteinte de leur site. Les coûts de protection étant bien souvent élevés, il peut être décidé de laisser venir la crue et d'arrêter l'activité au dernier moment.

La nécessité de disposer d'information sur la crue longtemps à l'avance revient comme un souhait récurrent au regard de la prévention et de la limitation des dommages certaines décisions sont en effet envisageables si un délai d'action existe pour acheter des groupes électrogènes, des matériaux de protection (parpaings, résines, ballons pour obstruer les collecteurs), des équipements de lutte contre la submersion (pompes).

La gestion du système de production dans l'optique d'une limitation des dommages dépend également de ce délai de réaction. Si l'activité est de type flux tendus, continue en trois-huit, il sera de toute façon très difficile de reporter la production dans le temps ; en revanche un report sur un autre site n'est pas à exclure si il est possible de l'organiser à temps.

Il est apparemment plus facile pour les sièges administratifs et commerciaux de se déplacer, mais la centralisation des informations attachée aux fonctions de ces sièges pose problème : comment transférer sur d'autres sites la comptabilité, les fonctions de surveillance et de contrôle d'autres établissements, etc.

\section{III — LA VALORISATION DES DOMMAGES INDIRECTS}

\section{- 3.1 La caractérisation monétaire de l'endommage- ment : les courbes de dommages}

La simulation de crues de périodes de retour différentes impose, pour l'évaluation des dommages, la construction de courbes reliant les paramètres hydrauliques d'une part (hauteur et durée de submersion) aux dommages correspondant à ces paramètres d'autre part. On constate, s'agissant des activités, d'importants effets de seuil conduisant à des écarts considérables en termes de dommages monétaires. Un des buts essentiels des enquêtes auprès des entreprises est d'ailleurs de dégager ces seuils.

L'exploitation des enquêtes en cours conduira à établir une courbe de dommages spécifiques à chacune des grandes entreprises situées dans l'enveloppe de la crue de 1910 pour

NOMENCLATURE DES ACTIVITES PROFESSIONNELLES (NAP) source: INSEE

\begin{tabular}{|c|c|c|c|}
\hline $\begin{array}{l}\text { NAP } \\
40\end{array}$ & INTITULÉ & $\begin{array}{l}\text { NAP } \\
40\end{array}$ & INTITULÉ \\
\hline 1 & agriculture, sylviculture, pêche & 20 & industries du bois, ameublement, divers \\
\hline 2 & industries de la viande et du lait & 21 & industries du papier et du carton \\
\hline 3 & autres industries agricoles et alimentaires & 22 & imprimerie, presse, édition \\
\hline 4 & prod. combustibles minéraux et cokéfaction & 23 & ind. du caoutchouc et transfo. mat. plastiques \\
\hline 5 & prod. pétrole et gaz naturel & 24 & ind. bâtiment, génie civil et agricole \\
\hline 6 & prod. et distrib. électricité, gaz et eau & 25 & commerce de gros alimentaire \\
\hline 7 & prod. de minerais, métaux férreux, transfo. acier & 26 & commerce de gros non alimentaire \\
\hline 8 & prod de minerais, métaux non ferreux & 27 & commerce de détail alimentaire \\
\hline 9 & prod matériaux construction, minéraux divers & 28 & commerce de détail non alimentaire \\
\hline 10 & industrie du verre & 29 & réparation et commerce de l'automobile \\
\hline 11 & chimie de base, prod. fibres synthétiques & 30 & hôtels, cafés, restaurants \\
\hline 12 & parachimie et industrie pharmaceutique & 31 & transports \\
\hline 13 & fonderie et travail des métaux & 32 & télécommunications et postes \\
\hline 14 & construction mécanique & 33 & services marchands aux entreprises \\
\hline 15 & construction électrique et électronique & 34 & services marchands aux particuliers \\
\hline 16 & construction automobile, transports terrestres & 35 & location et crédit bail immobiliers \\
\hline 17 & construction navale, aéronautique et armement & 36 & assurances \\
\hline 18 & industries textile et de l'habillement & 37 & organismes financiers \\
\hline 19 & industries du cuir et de la chaussure & 38 & services non marchands \\
\hline
\end{tabular}


la région Ile de France. On pourra ainsi valider les estimations antérieures sur une base statistique nettement plus fiable. A ce stade des investigations, il semble acquis que les ordres de grandeurs antérieurs (plusieurs milliards de francs pour les dommages indirects aux activités) ne seront pas infirmés.

Pour les activités non enquêtées, une distinction sera établie entre activités de production, de stockage et de négoce. Les investigations auprès des experts assurantiels devraient aider à élaborer des endommagements spécifiques à ces types d'activités. Il est indispensable de parvenir à différencier les activités en tenant compte de la part de production récupérable et du délai de retour à la production normale.

\section{- 3.2 Effets multiplicateurs et indirects de la crue}

Une crue de type 1910 à Paris et dans la région lle de France est typique de ce que l'on qualifie familièrement de “ scénario catastrophe ". L'étendue de la zone inondable, incluant un milieu urbain dense, induit un effet multiplicateur sur les dommages individuels aux entreprises, par accumulation des contraintes : coupures des voies d'accès, interruption de prestations de services publics (transports, énergie, télécommunications). Dans ces conditions, le maintien de la production ou son transfert deviennent encore plus aléatoires.

Par ailleurs l'intégration d'une activité donnée dans une chaîne donne lieu à des effets indirects ou effets " en cascade ". C'est le cas bien connu des grands groupes industriels dans leurs relations avec leurs sous-traitants.

Les pertes de production futures sont quelquefois difficiles à quantifier : la perte de clientèle d'un restaurant à la suite d'une fermeture de plusieurs mois, par exemple ; ou bien des dommages se manifestant longtemps après l'événement du fait de l'usure accélérée de certains éléments du système physique de production (cablages, éléments en bois).

Enfin les impacts humains, psychologiques et sociaux, même s'ils ne sont pas toujours aisément quantifiables, ne sauraient être négligés dans ce type de scénario. Dans toute catastrophe naturelle, l'homme reste le centre du débat. 